\title{
EL PROBLEMA DE LA BARRERA PSICOSOCIOCULTURAL INTERPUESTA ENTRE LOS PROFESIONALES DE LA SALUD Y CONSULTANTES DE CONTEXTOS POBRES ESTRUCTURALES. REFLEXIONES SOBRE UNA CUESTIÓN COMPLEJA THE PROBLEM OF PSYCHOSOCIOCULTURAL BARRIER INTERPOSED BETWEEN HEALTH PROFESSIONALS AND CONSULTANTS FROM STRUCTURAL POOR CONTEXTS. REFLECTIONS ON A COMPLEX ISSUE
}

Recibido: 07 de Agosto del 2013 | Aceptado: 26 de Noviembre del 2013

\author{
MARÍA PAULA JUÁREZ $Z_{1}$ ENRIQUE SAFORCADA 2 \\ (UNIVERSIDAD NACIONAL DEL RÍO CUARTO, Córdoba, Argentina)
}

\section{RESUMEN}

El artículo analiza la dinámica del proceso en función del cual se genera la barrera psicosociocultural y se le da soporte de sostenibilidad en el ámbito de las relaciones entre médicos/médicas pediatras y madres consultantes residentes en contextos pobres estructurales (CCPE). Se ahonda en el concepto de barrera psicosociocultural y su estructuración; se analizan comportamientos intersubjetivos (pediatras / madres) y sus consecuentes reacciones afectivo-emocionales que activan esta barrera. Se vincula este proceso con el concepto de extensión en la pedagogía de Paulo Freire y se reflexiona sobre la importancia de la formación universitaria de los profesionales de la salud, en pregrado y posgrado, como el factor principal en la constitución y mantenimiento del mencionado obstáculo que tiene una fuerte incidencia en la eficacia y eficiencia de la inversión del Estado en el sector salud. Se hace hincapié en la necesidad de transformaciones curriculares de la carrera de medicina como instancia que posibilitaría la formación de un profesional con un perfil relativista cultural, dialógico y prosocial que llevaría a la desactivación de los procesos y dinámicas que esterilizan el accionar facultativo en salud.

PALABRAS CLAVE: Barrera psicosociocultural, consultantes de contextos pobres estructurales, formación médica.

\section{ABSTRACT}

The article analyzes the dynamics of the process by which the psycho-socio-cultural barrier between pediatricians and patient mothers living in poor structural contexts is generated and sustained. It delves into the concept and structure of the psychosocio-cultural barrier; it analyzes inter-subjective (pediatrician/mother) behaviors and the resulting affective-emotional reactions which activate this barrier. This process is linked with the concept of extension in the pedagogy of Paulo Freire and reflects the importance of the university education of health professionals, both undergraduate and graduate, as the main factor in the formation and maintenance of this obstacle which has a strong impact on the effectiveness and efficiency of state investment in the health sector. It emphasizes the need for curricular changes in medical training as a means encouraging the formation of professionals with culturally relevant, conversational and sociable profiles, which in turn would lead to the cancelling of the processes and dynamics which impede useful action in medicine.

KEY WORDS: Psycho-socio-cultural barrier, patient mothers, poor structural contexts, medical education.

1. Doctora, Universidad Nacional de Río Cuarto, Consejo Nacional de Investigaciones Científicas y Técnicas (CONICET) Córdoba, Argentina. E-mail: mpaulajuarez@gmail.com. La correspondencia deberá ser dirigida a la Dra. María Paula Juárez.

2. Doctor, Universidad de Buenos Aires - Universidad Nacional de Avellaneda. Buenos Aires, Argentina. E-mail: enrique.saforcada@gmail.com 


\section{INTRODUCCIÓN}

La lectura de la realidad social e histórica del contacto interactivo, por un lado, entre agentes sociales de niveles medios socioeconómicos preparados para el desempeño de roles caracterizados al servicio del desarrollo humano, como es el caso de los profesionales de la salud y, por el otro, las poblaciones afectadas por la pobreza estructural generada por una determinada dinámica y dispositivos económico políticos, interpela una reflexión crítica al tiempo que exige una alternativa superadora inmediata.

Desde hace varios años realizamos investigaciones y experiencias de campo basadas en el paradigma de salud comunitaria (Saforcada, 2001, 2011; Juárez, 2009a, 2009b, 2012) las que nos permitieron conocer la realidad de diversos grupos de vecinas y vecinos de comunidades pobres residentes en las provincias de Buenos Aires y Córdoba, Argentina.

Éstas permitieron visibilizar una
roblemática común, emergente y seguramente advertible en diversos lugares. Se trata de dificultades de relación, interacción y comunicación entre miembros del componente formal del sub-sistema de servicios públicos de salud y miembros del componente informal de estos servicios: médicos-médicas / vecinos-vecinas consultantes de contextos pobres estructurales (CCPE). Encuentros, o mejor dicho, "desencuentros" donde se manifiestan, principalmente por parte de los profesionales hacia los consultantes, situaciones de extensión comunicativa, cosificación, menosprecio de la palabra del otro, actitudes de rechazo, valoraciones descalificantes, desconfianza, incomprensión, imposición de significado desde el uso exacerbado de léxico científico, entre otras.
Consideramos que estos escenarios de antidialoguicidad, al decir del pedagogo brasileño Paulo Freire, entre las comunidades y los profesionales de la salud, propician el establecimiento de una barrera psicosociocultural cuyo correlato es, no sólo la deshumanización de los encuentros, sino el impacto negativo que ello tiene en el perfil que constituye a las personas que se encuentran en situación de pobreza estructural. Al reflexionar e investigar sobre este problema, responsable de un porcentaje considerable de las causas de la ineficacia e ineficiencia de la inversión de recursos en salud por parte del Estado, se debe tener en cuenta que la relación de poder entre efectores y consultantes de un servicio público de salud es profundamente desigual: el poder profesional en el campo de la salud agobia las posibilidades de autoafirmación de los pobres y tiene una gran capacidad, potencial o actual, para dañar su autoestima e incrementar, entre otros aspectos, la indefensión aprendida que, en términos generales, caracteriza a estas poblaciones.

Intentando problematizarnos sobre la complejidad del "desencuentro" mencionado, nos preguntamos: ¿cuáles serían algunos comportamientos propiciados por parte de los y las profesionales de la salud que dinamizan el funcionamiento y estructuración de la barrera psicosociocultural hacia los y las consultantes de contextos pobres estructurales (CCPE)? ¿Cómo impacta la formación universitaria recibida por los y las profesionales en la configuración de contenidos representacionales $y$ comportamentales que propician esta barrera? ¿Qué futuro es dable pensar para esta relación y su impacto psicosociocultural?

Atendiendo a lo planteado, el propósito de este artículo es compartir algunos lineamientos y dispositivos conceptuales orientados a contribuir a la comprensión del papel que desempeñan los profesionales de 
la salud en la estructuración y dinamización de la barrera mencionada al interactuar con CCPE. Para ello intentaremos: 1) plantear una discusión teórica que nos permita definir qué entendemos por barrera psicosociocultural y su estructuración; 2) desarrollar brevemente el enfoque metodológico que utilizamos para conocer dicha barrera; 3) analizar y reflexionar sobre los resultados hallados en torno a algunos comportamientos afectivo-emocionales de los profesionales que activan esta barrera y la formación universitaria de los profesionales como un factor configurador de los contenidos de sus concepciones psicosocioculturales que intensifican la barrera cuando entran en contacto con CCPE. Finalmente, se realizarán las conclusiones del trabajo y se intentará avanzar sobre una propuesta que contemple el horizonte de la complejidad de dicha relación y la posibilidad de desactivación de los procesos y dinámicas que dan origen a la barrera misma.

\section{DISCUSIÓN TEÓRICA}

El concepto de barrera psicosociocultural y su emergencia en el encuentro entre profesionales de la salud y consultantes de contextos pobres estructurales

Diversos autores han analizado las problemáticas que caracterizan al encuentro entre médicos y consultantes, éstos han denominado la situación de múltiples maneras, tales como: vinculación inquietante (Ferrara, 1985); encuentro insatisfecho (Thompson 1990), choque de creencias (Kalinsky y Arrúe 1996); dualidad vincular de tinte verticalista (Emmanuelle, 1998); relación inclusiva de desigualdad social (Mendoza González, 1999); barreras culturales y de género (Lasso Toro, 2012), tensiones 0 desencuentros (Lasso Toro, 2012).

Asimismo, distintas subdisciplinas han realizado abordajes a dicha problemática: la psicología médica, la psicología sanitaria (Saforcada, 2001, 2002a; de Lellís, Álvarez,
Rosetto y Saforcada, 2006; Saforcada, de Lellís y Mozobancyk, 2010), la psicología de la salud (Morales Calatayud, 1999) y la comunicación en salud (Feldman-Stewart, Brundage, y Tishelman, 2005; Juárez 2009b; Escotorin, Juárez, Cirera y Roche, 2011; Vidal y Benito, 2010), entre otras.

En este trabajo se aplica el concepto de barrera psicosociocultural. Lo definimos como un fenómeno que resulta de la estructuración, en un espacio intersubjetivo virtual, de los efectos de las reacciones afectivo-emocionales negativas producto de los comportamientos interaccionales de las personas que integran el componente formal de los sistemas de servicios de salud (personal de hospitales, centros periféricos, etc.) y las personas que constituyen el componente informal de estos servicios (las poblaciones que acuden a estas instituciones en busca de respuestas a sus problemas de salud, percibidos como tales por ellas mismas).

Este espacio intersubjetivo virtual se ubica, esquemáticamente, entre ambos componentes (formal e informal) y en el se establece la mencionada barrera, la cual no es tangible fácilmente -no está activa, ni es visible ni se interpone en forma continuadado que solo se la puede percibir cuando las subjetividades de uno y otro componente entran en interacción y se observa atentamente el proceso implicado. Otra alternativa para detectarla es la investigación psicosocial específicamente dirigida a este fin.

De modo indirecto se puede descubrir su presencia a través de la búsqueda estéril de explicación a la habitual falta de eficacia (recidivas inesperadas -la mayoría de ellas, falta de adhesión a las prescripciones facultativas 0 tratamientos, etc.) y/o a la también frecuente presencia de ineficiencia inexplicable (distorsiones en la relación inversión / resultados, manifestaciones políticas de crítica por parte de las poblaciones, capacidad instalada en el área 
insuficiente, entre otros) de los sistemas de servicios de salud.

La barrera psicosociocultural es siempre obstaculizadora y distorsionadora de una interacción que -desde lo racional y el respeto por los derechos humanos- debería llevar al siguiente tipo de resultados:

- En la perspectiva del componente formal: lograr el mayor bienestar posible de las personas consultantes, la confirmación de sus autoestimas y el logro de las metas buscadas por esta interacción 0 implícitas en ella: mantener sanas a las personas sanas, prevenir el daño amenazante a la salud, curar tempranamente el daño instalado y restablecer, cuando se diera tal situación, las capacidades perdidas por el daño (secuelas).

- En la perspectiva del componente informal: mantener y aumentar si es posible, 0 alcanzar o recuperar el bienestar dependiente de la condiciones de salud autopercibidas.

Estos resultados se alcanzan cuando en lugar de una barrera psicosociocultural se estructura un puente facilitador de la interacción, el cual es producto de vivencias positivas implicadas en las reacciones afectivo-emocionales surgidas de la interacción entre profesionales efectores y pobladores consultantes. Se llega a esta alternativa cuando el espacio interactivo tiene como soporte la consideración permanente de que quienes interactúan integrantes del componente formal e integrantes de la población- son sujetos de derechos integrales inalienables y esos derechos son respetados cotidianamente por ambas partes.

¿Qué le da permanencia, aunque latente, a esta barrera y se la da o daría a un puente facilitador? El hecho de que las mencionadas reacciones afectivo- emocionales negativas 0 positivas se transforman en, 0 son germen de, la estructuración (en la subjetividad de las personas interactuantes) de representaciones sociales, sistemas valorativo-actitudinales, sistemas de atribución, creencias, visualizaciones, opiniones, y otras formas de elaboración psicosocial de las vivencias, a través de las cuales los seres humanos perciben $y$ significan el mundo circundante -en este caso, los ámbitos de la salud- a la vez que regulan sus comportamientos y los significados de los mismos. Estas estructuraciones, al cabo de un tiempo y de su generalización en las poblaciones, terminan por incorporarse a las estructuras subculturales implicadas: las subculturas institucionales de los sistemas de servicios de salud y las subculturas sociales, particularmente las propias de los y las consultantes de contextos pobres estructurales que son quienes mayoritariamente concurren a los subsistemas públicos de servicios de salud.

¿Por qué son negativas en el caso de la barrera?, porque implican formas diversas de descalificación del otro, de desvalorización, de irrespeto de los derechos humanos de la otra persona, con lo cual se vulneran e insatisfacen muchas de las necesidades de autorrealización, reconocimiento, afiliación y seguridad que señala Maslow (1991) en su teoría de las necesidades humanas. ¿Y por qué son positivas en la alternativa del puente?, porque satisfacen estas necesidades.

El tema de las necesidades también es de mucho valor analizarlo desde la perspectiva de Max-Neef, Elizalde y Hopenhayn (1986) que, además, es una fértil construcción de teoría local regional, específicamente en Chile. En este desarrollo las necesidades humanas son agrupadas en dos dimensiones: necesidades según categorías existenciales -señalan cuatro- y necesidades según categorías axiológicas -señalan nueve, lo 
que da mayor riqueza a las reflexiones e investigaciones que se hagan sobre este problema de la barrera como obstaculizadora de la satisfacción de necesidades y del puente como facilitador.

Se considera a la pobreza como producto de decisiones, acciones y omisiones políticas e, indirectamente, del enfrentamiento de clases. Estableciendo una diferenciación cualitativa, podrían advertirse dos grandes categorías de situaciones de pobreza:

- Aquellas situaciones de pobreza que son reversibles con solo otorgar el dinero suficiente para que personas, familias y comunidades puedan adquirir los medios por ellos conocidos para comenzar a vivir con estilos propios de quienes no son pobres 0 no se autoperciben como pobres.

- Otras situaciones de pobreza que generan condiciones tales que la presencia solo del dinero no permite a las personas 0 familias salir de esta condición y, generalmente, profundiza 0 agrava los aspectos negativos de tales condiciones de vida.

A estos pobres a los que el dinero no saca del estilo de vida de la pobreza extrema y que, por el contrario, si les llega en forma directa, en general los perjudica, es lo que denominamos pobres estructurales. La pobreza estructural se caracteriza por un conjunto de indicadores que configuran una entidad polimórfica y extremadamente grave, entendiendo por gravedad no la cercanía 0 alta probabilidad de muerte sino la abundancia de daños (biológicos, biopsicológicos, psicosocioculturales, etc.) y sufrimiento. Es una problemática compleja que se reproduce con progresivo agravamiento a través de la dinámica familiar y la interacción de la familia con el ambiente en donde reside, generándose un particular estilo de vida que obstaculiza el desarrollo pleno del potencial genético (Saforcada et al., 2010, pág. 238).

En los planos biológico y neuropsicológico se observa desnutrición y problemas asociados, o sea defectos en el crecimiento y desarrollo, falta de estimulación sensorial y afectivoemocional, con el consiguiente efecto negativo en el neurodesarrollo que se suma al proveniente de las carencias alimentarias $y$, frecuentemente, a la contaminación ambiental.

En el plano psicosocial se manifiesta en un conjunto relevante de indicadores 0 síntomas: a) desaliento y desesperanza; b) defraudación, entendida como el sentimiento generado por la estafa de gestión en cuanto a lo prometido por los políticos y las figuras que pretenden llegar al poder, promesas que luego son ignoradas; c) los sentimientos de discriminación; d) comportamientos de abandono; e) muy baja autoestima y autoimagen negativa; f) desarrollo temprano de la indefensión aprendida (Seligman,1989); g) locus de control totalmente externo; $h$ ) desarrollo de comportamientos y destrezas noadaptativos; i) bloqueo del aprendizaje de destrezas adaptativas.

En el plano psicocomunitario emergen indicadores tales como: a) imposibilidad de generar proyectos de vida adaptativos; b) alta tasa de consumo y adicción de sustancias psicoactivas en los varones; $c$ ) alta tasa de embarazo adolescente.

Atendiendo a lo planteado, es razonable pensar que la barrera psicosociocultural indeliberadamente generada por profesionales, estudiantes y practicantes de las ciencias de la salud- dinamiza un conjunto de reacciones afectivoemocionales y componentes axiológicos, representacionales, actitudinales y comportamentales que coadyuvan a reforzar los indicadores 0 síntomas psicosociales que integran el perfil 
nosográfico de la pobreza estructural. Entre ellos se pueden señalar la baja autoestima, el síndrome de indefensión aprendida, la autodiscriminación y el abandono, el miedo social, la defraudación, el desaliento y la desesperanza, entre otros.

Esta barrera es producto de una dinámica sistémica en que cierto tipo de interacción entre consultante y profesionalefector genera reacciones afectivoemocionales que van a retroalimentar las estructuras valorativo-actitudinales $y$ representacionales preexistentes en el componente informal (pobladores) así como en el componente formal (personal del Subsistema Público de Servicios de Salud).

Esta característica (sistémica y retroalimentativa) transforma a la cuestión de la barrera psicosociocultural no en un círculo vicioso sino en un espiral vicioso, a medida que pasa el tiempo sin que intervengan acciones de atenuación 0 solución, el problema irá en aumento: profesionales progresivamente más anómicos y desmoralizados por un lado $y$, por otro, población progresivamente consolidada psicosocialmente cristalizada en su condición de pobres estructurales que también, de modo permanente, van derivando a la automedicación, los remedios caseros, las prácticas que vinculan con lo sobrenatural (Gauchito Gil, Difunta Correa, la escobita de San Martín de Porres, la cruz de Caravaca, etc.) y las otras formas no profesionales de atención de los problemas de salud con quienes no se ven discriminados (efectores que apelan a lo sobrenatural y curadores populares).

\section{METODOLOGÍA}

Para indagar en la problemática de la barrera psicosociocultural se realizó un estudio exploratorio que permitió conocer la complejidad de la interacción y comunicación en el marco de la relación médico-médica/ -madre consultante (Juárez, 2012).
Se empleó la estrategia metodológica del estudio instrumental y colectivo de casos, complementada con otros métodos cualitativos y orientados hacia la construcción conceptual (Stake, 1998; Neiman y Quaranta, 2006).

La unidad del estudio se definió como las entrevistas de consulta que mantienen médicos y médicas pediatras con madres consultantes. Los casos se definieron atendiendo a los médicos y médicas por ser la figura de la relación que se mantiene constante, a diferencia de los niños y madres que se renuevan entre consultas.

Para la selección de los casos se utilizó un criterio pragmático (Hammersley y Atkinson, 1994) que permitió escoger, de un amplio grupo de médicos y médicas que se desempeñan en el Primer Nivel de Atención del Sub-sistema Público de un municipio de la ciudad de Córdoba (Argentina) a aquellos que estarían dispuestos a ser observados, definiéndose la muestra por cuatro casos: dos médicas pediatras y dos médicos pediatras. Éstos fueron estudiados por un período de tres meses en jornadas de observación con una frecuencia semanal por el transcurso de dos horas en su desempeño de atención de la salud. Los instrumentos de recolección de datos utilizados fueron observación, entrevistas individuales y una entrevista colectiva, estrategias que se utilizaron conjuntamente.

La retirada del campo se realizó cuando se produjo la saturación de los datos lo que evidenció que la nueva información comenzaba a resultar poco provechosa dada su reiteración (Strauss y Corbin, 1998).

Dado el gran volumen de datos recabados (treinta y siete documentos) para su sistematización global, codificación y recuperación para su análisis integral se empleó del programa informático Atlas-TI versión 2.4 . 
Para su análisis se procedió desde los planteos de la Teoría Fundada orientados por una primera etapa de codificación abierta, seguidos por una codificación axial y selectiva, efectuando durante estos procesos comparaciones de manera constante, todo lo que nos permitió identificar categorías, desarrollarlas, relacionarlas entre sí e ir comprendiendo los datos examinados en relación a los casos (Strauss y Corbin, 1998).

\section{RESULTADOS}

Atendiendo a lo desarrollado algunos de los ejes temáticos emergentes del estudio se vinculan a: comportamientos afectivoemocionales de profesionales de la salud hacia consultantes, la asimetría y el ejercicio de poder como reacciones de los profesionales y contenidos de las estructuras psicosocioculturales de los profesionales de la salud que dinamizan la barrera. Aspectos que desarrollaremos a continuación.

\section{Comportamientos afectivo-emocionales de profesionales de la salud hacia consultantes de contextos pobres estructurales que generan y refuerzan la barrera psicosociocultural: el caso de la extensión}

Entendemos por comportamientos afectivoemocionales a las respuestas psicoafectivas, comportamentales y actitudinales, que se generan en las interacciones entre los consultantes y los profesionales de la medicina.

Asumiendo la perspectiva pedagógicocritica de Paulo Freire, que abona el actual paradigma de salud comunitaria (Saforcada et al., 2010), tomamos la denominación freireana de "extensión" (Freire, 1973) para señalar un tipo particular de comportamientos profesionales que generan en el otro $u$ otra (CCPE) reacciones afectivo-emocionales (negativas en este caso) que van, por un lado, a estructurar y reforzar la barrera psicosociocultural $y$, por otro, a integrar en la subjetividad de las y los consultantes, los componentes que configuran las estructuras de significaciónvaloración que regulan los comportamientos humanos y la autopercepción. En este tipo de interacción el profesional impone su figura, sus saberes y concepciones a la persona del CCPE, a quien no respeta en su dignidad y del que no espera respuestas de retroalimentación cognitiva.

Primordialmente en el plano comunicacional se trata de explicaciones (sean indagaciones anamnésicas, diagnósticos o tratamientos, indicaciones, prescripciones) realizadas mediante verbalizaciones "depositadas" de manera breve, efímera, rápida, o compleja, desde una actitud y comportamiento de imposición y distanciamiento subcultural que perjudica la comprensión del CCPE por una vía de impacto directo que proviene de cuestiones semánticas y por una vía indirecta vinculada con la emoción que afecta asuntos tales como la autoestima, los sentimientos de inferioridad reforzando fenómenos tales como la indefensión aprendida.

Cuando en la relación se dinamiza la extensión como comportamientos del profesional hacia el CCPE lo que predomina son manifestaciones de: a) imposición de significados (que dan cuenta de incomprensión y falta de empatía hacia la figura del CCPE); b) cosificación (visible en valoraciones descalificantes, atribuciones desfavorables e imágenes negativas hacia la persona del CCPE); c) interrogatorio extensionista (denotando el poder de prescindir de la palabra del otro a través de actitudes de rechazo y desconfianza al CCPE ). Todo un escenario en el que los comportamientos y actitudes del profesional están guiados por un ejercicio de poder exacerbado y autoritario (el profesional autocrático escucha y finalmente decide por sí, el autoritario ni siquiera escucha).

La extensión como comportamiento del profesional que propicia la barrera opera imposibilitando la comunicación y 
generando asimetría dado que se emplea un sistema simbólico ininteligible para los sujetos CCPE, imperando un lenguaje técnico en relación a un hecho con un universo de signos lingüísticos propios que son mal comprendidos por los consultantes interlocutores.

Freire (1973) considera que la extensión supone un sentido de superioridad con que los profesionales se enfrentan a las personas concebidas como "cosa", como "objetos", como "inferiores", negándolos como seres de acción, de transformación del mundo. El que extiende deposita un conocimiento elaborado a los que no lo tienen, eliminando su capacidad crítica para tenerlo, irrumpiendo en su espacio históricocultural distinto, ignorando sus potencialidades, su creatividad e inhibiendo su expansión.

La extensión en todas sus modalidades de impacto afectivo-emocional (violencia de significado, cosificación de consultantes, interrogatorios extensionistas) puede advertirse como un estilo de desempeño de rol, personal e interactivo, así como una especie de forma relacional de algunos médicos y médicas. A continuación se consignan algunos ejemplos de estos comportamientos hallados en un estudio anterior sobre la relación entre médicos/médicas pediatras y madres CCPE (Juárez, 2012):

A. La imposición de significado. Se trata de un comportamiento profesional en el que prima el empleo excesivo de un lenguaje académicocientífico que genera un choque con el lenguaje popular de los consultantes que sufren un menoscabo a su forma de comunicación habitual, resultando como consecuencia, muchas veces, situaciones de violencia de significado propiciada por el profesional. Algunas narrativas que permiten graficar que se entiende por imposición de significado expresan:

(Escenario de consulta con dos madres bolivianas)

C4: (...) al no tener el certificado de nacimiento eso es una traba que te pone el registro civil entonces vamos a tener que hacer con la asistente social una serie de pasos para que tenga su documento, si no, no va a tener documento $\mathrm{y}$ por supuesto que el documento es muy importante que lo tenga, $y$ aparte vamos a ver si hacemos unos análisis en el hospital también...

Cuñada (acompañante de la madre consultante): ¿ese de hipotiroidismo tiene que hacerle a ella?

C4: y... (suspira) ese tendría que hacerlo también, yo te lo tengo que pedir a ese "screening neonatal", eso lo tendrías que hacer en maternidad, en maternidad 0 en el hospital... (122:133. P29:25. $1^{\circ}$ Observ. C4).

Es frecuente observar en las consultas el empleo de términos que a los profesionales les resultan corrientes y al emplearlos en la práctica, en supuesto diálogo con los consultantes, se advierte que estos no son términos comunes al universo de sus significados, y lo que se pretende como comunicativo termina resultando en imposición de significados. La lectura de esta narrativa lleva a preguntar ¿sabrá la madre boliviana a que se refiere la médica con screening neonatal? Muy probablemente desconozca su significado, siendo necesario explicárselo a la madre por parte de la médica.

Ma2: no, le quería preguntar a usted por el tema del bajo peso, doctor 
C1: ipero no está bajo peso!

Ma2: ahora ya subió porque el mes pasado...

C1: (la interrumpe) ino pero no

está bajo peso!... esta en un

"peso bajo", pero no es "bajo peso"

Ma2: ah...

C1: ¿me explico?

Ma2: no, sí, sí pero como venia

todos los meses (739:759.

P5:113. $4^{\circ}$ Observ. C1).

Probablemente esta madre no haya disipado su duda con esa respuesta que quizás para el médico es clara desde su conocimiento de taxonomías de peso y talla para el crecimiento infantil, y que para la madre puede resultar como una especie de juego confuso de significados, no logrando aclarar su incertidumbre dado que el profesional no le ofrece una diferenciación detallada de lo que se entiende por "peso bajo" y "bajo peso". Si bien el médico parece intentar cerciorarse de que la madre ha comprendido al preguntar " $¿ m e$ explico?", ella inmediatamente responde "no, sí, si", como una respuesta mecánica a una explicación mecánica que ha prescindido de los elementos dialógicos necesarios para la comprensión de lo dicho.

B. Cosificación de los consultantes. Es el comportamiento en que el médico/médica se relaciona con el niño/niña y la madre consultante en tanto objetos (no sujetos) de su estudio. Su comunicación verbal con ellos es prácticamente nula dado que no se ofrecen explicaciones del cuadro, no se esfuerza por emplear tonos de voz que propicien la escucha, el médico/médica mismo no escucha, no pregunta, ni tampoco considera los interrogantes, dudas 0 indagaciones de su consultante. Hace caso omiso de la palabra de la consultante, la que descalifica, desestima, desacredita, menosprecia; sumado a una actitud de evadir el contacto cara a cara con sus consultantes, respecto a los que, muy probablemente, sólo ha configurado imágenes negativas y atribuciones desfavorables.

Algunas narrativas que podrían dar cuenta de reacciones de cosificación a consultantes son de la siguiente modalidad:

El médico habla bajo pero además
denotando poco entusiasmo para
hacerlo, obligando a que la madre de
su paciente haga un esfuerzo
notable para escucharlo. El médico
se sienta a rellenar planillas y
escribir recetas y ordenes. Silencio.
Le entrega a la madre las 6
prescripciones o recetas de cosas a
hacer, 6 papeles distintos que le
entrega uno detrás de otro
diciéndole brevemente que hacer
con cada uno, ella denota esfuerzo
para recordar qué es cada uno, pero
imagino que al llegar a su casa
puede haber tenido dificultad para
hacerlo de la manera
correcta.(32:41. P2:104. $1^{0}$ Observ.
C1).

La actitud de concentración en que muchos profesionales suelen mostrarse disfraza una real introversión vinculada a la antidialoguicidad desde la cual algunos médicos/médicas se desempeñan. El permanecer ensimismado, absorto en un mundo propio mientras se comparte con el consultante un espacio que se supone de intercambio activo necesario es una forma de cosificar a los CCPE, de reducirlos a la concepción de objetos. El hablar en un tono de voz imperceptible, el desenvolverse indiferente a la presencia del otro en el espacio común, el permanecer inmutable y el depositar prescripciones sin mediar palabra o hacerlo desde la verborragia que abruma y no comunica, es un ejemplo concreto de extensión en su modalidad cosificadora. Con la negación a la escucha a la madre consultante el médico reacciona 
cosificándola, niega su interrogante quitándole importancia. De igual manera se cosifica a la madre en la medida que no se la considera como fuente de indagación con la cual intercambiar significados comunes del caso, sino que se le extienden enunciados interrogativos desde el empleo de modismos verbales y tonos de voz que parecen inculparla. También hay cosificación en la medida que el profesional formula una pregunta que la madre no contesta porque no comprende y el médico no se esfuerza en volver a preguntar para ser entendido por ella.

C1: a ver acostalo, a ver cómo está la garganta.... Esa tos ¿es con.... con secreciones? O... ¿hizo secreciones? -tenele la cabezaMa8: (silencio parece no entender lo que le dice)

C1: (no vuelve a preguntar $y$ continúa el control) (1877:1881. P3:114. $2^{\circ}$ Observ. C1).

Ma4: (alza la niña a la camilla)

$\mathrm{N}$ : (llora)

A: jabrí la boca!

C1: jtenele la cabecita! (le ve la boca) iya está!

Ma4: ¡ya está viste!

C1: (se sienta a escribir las prescripciones, silencio, solo dialogan enfermera $y$ administrativa)

Ma4: que es lo que... ¿qué remedio le va a dar doctor?

C1: (no le responde... silencio prolongado)

Ma4: (asoma la cabeza para leer prescripciones) ¿nebulizaciones nada?

C1: ipara un segundito mamá! (eleva el tono de voz, continúa escribiendo prescripciones)

Ma4: a bueno...

C1: (continúa escribiendo prescripciones) (1113:1148.

P5:59. $4^{\circ}$ Observ. C1).
En estas narrativas toda posibilidad de diálogo se diluye al no mostrarse el profesional abierto a la comunicación siendo incapaz de reformular enunciados, preguntas 0 indagaciones para ser interpretado por las madres CCPE.

C. Interrogatorio extensionista. Se trata de un comportamiento profesional que da cuenta de una indagación realizada al consultante por medio de preguntas pautadas, estandarizadas, sobre el cuadro por el que se consulta, caracterizada por carecer de profundidad y prescindir de la escucha como elemento clave en la construcción del significado del cuadro presente.

Se trata de una indagación normatizada, casi esquemática 0 pautada, que se realiza como un paso formal y rutinario de la práctica médica de atención que implica trabajar siempre ante un paciente tipo, excluyendo las inevitables características personales del enfermar. No se indaga en profundidad y se prescinde de la escucha como elemento para la construcción del significado de lo que acontece (que es lo que conduce al diagnóstico acertado) ya que se considera de antemano que por esa vía no se podrá acceder al conocimiento de la situación. La idea subyacente al interrogatorio extensionista es que el cuerpo, como objeto de conocimiento, es el que revelará las claves o elementos para descifrar el cuadro por el que se consulta, pudiendo prescindir el médico de las narrativas de los sujetos. El interrogatorio se realiza como un paso más desde un habla impositiva.

\section{C1: ¿Qué come? (indagación} médica) 
Ma5: puré... fideos (respuesta de la madre)

C1: ¿puré de qué? (indagación médica)

Ma5: carne... (respuesta de la madre)

C1: (interrumpe y eleva el tono de voz) ¿puré de qué madre? (ausencia de una escucha profunda previa, ausencia de tolerancia a los tiempos del consultante. Negación a sus contenidos expresados)

Ma5: de zapallo, zanahoria... toma agua... eh come yogurt (respuesta de la madre)

C1: ¿y leche? (indagación médica)

Ma5: leche también... (1469:1491. P5:66. 40 Observ. C1).

La actitud asumida por el profesional que efectúa un interrogatorio extensionista está caracterizada por la intolerancia, la desconfianza y el rechazo a la figura del CCPE, junto con la impaciencia, la autosuficiencia, el hacer notar al consultante la brevedad de tiempo disponible para su atención, condicionando una limitación a sus narrativas. Muchas veces, con estos comportamientos, los médicos suelen denotar un talante desganado a la escucha considerándola una pérdida de tiempo, interrogan repitiendo varias veces las mismas preguntas dando cuenta de la ausencia de una escucha profunda, sin aguardar los tiempos necesarios para las respuestas de la consultante, guiando el interrogatorio de acuerdo a sus suposiciones y con un objetivo predeterminado.

2. El denotar asimetría y ejercer poder como reacciones de los profesionales que propician la barrera

Los comportamientos afectivo-emocionales en que se manifiesta la extensión antidialógica se ven propiciados por un marco asimétrico en el que se evidencia ejercicio de poder.

Cuánto más asimétricas son las características culturales de los interlocutores más se diferencia la relación, y cuando esta diferencia implica una relación de poder o autoridad o una cierta prevalencia culturalmente aceptada o impuesta, la relación que debiera ser complementaria se transforma en una relación jerárquica (Haley, citado en Izquierdo 1996, p.42). Entonces se distingue la llamada posición primaria 0 superior de un interlocutor (one-up) de la posición secundaria o inferior (one-down) que ocupa el miembro complementario que apela a la autoridad o acepta la prevalencia del interlocutor.

Consideramos que la cuestión del ejercicio del poder por parte del médico 0 médica en la comunicación e interacción con los CCPE no es sólo un aspecto sobre el que hay que trabajar a fin de exigir la obligación de respetar a las personas y todo lo que ello implica, sino que ese ejercicio de poder menoscabante del otro tiene efectos muy negativos en la autoestima de los y las consultantes y por lo tanto en su salud o en la evolución de sus enfermedades. Entendemos que afecta dos cuestiones básicas:

1. El trato autoritario genera rechazo y esto redundaría en aspectos que probablemente no operan en el plano consciente de los y las CCPE y terminan generando comportamientos de no adhesión a los tratamientos promoviendo, por lo contrario, la concurrencia 0 consulta a referentes populares en salud no profesionales, la recurrencia a remedios caseros, etcétera.

2. Efectos agravantes que pueden incidir en los y las CCPE generando problemas psiconeuroinmunoendocrinos (PNIE) que deprimen su sistema inmunológico, ponen en riesgo su salud y estado anímico. 
De acuerdo a Ferrara (1985) las condiciones sociales en las que se han desenvuelto las historias individuales y grupales de médicos/médicas, por un lado, y las de CCPE, por otro, forjan estas asimetrías como una forma de asignación de poder, como una exigencia para reafirmar la situación que se evidencia en la sociedad. A través de múltiples estrategias se han intentado modificar estas asimetrías, pero la situación sigue siendo igual e injusta ya que se encuentra enraizada y sometida por las realidades emergentes de las relaciones sociales, siendo la transformación de estas la única posibilidad de modificar las asimetrías.

Gwyn (2002) considera que deben estudiarse las interacciones en el cuidado de la salud a fin de deconstruir el conflicto de intereses entre médicos y consultantes, atendiendo a que los rasgos de las conversaciones entre ambos son consideradas como de inequitativa naturaleza en las relaciones de poder. La inequidad del poder es presentada como un hecho social, establecido; de acuerdo a Treichler et al., "un a priori en los participantes en la típica consulta" (Citado en Gwyn, 2002, p. 63).

El conglomerado de médicos/médicas, apoyado en sus corporaciones y en el marketing del complejo industrial que usufrutúa el mercado de la enfermedad, ejerce poder, expresa la hegemonía de su grupo, modela su influencia y marca la fuerza que, por un lado, le ha concedido la sociedad y, por otro, han construido con sus estrategias de dominación basadas, frecuentemente, en procesos de dominación cultural, establece y reafirma cotidianamente su jerarquía y de esa manera "acentúa la represión, volcada hacia los síntomas, a las causas aparentes, llevando al enfermo a una situación de dependencia en la que es interpretado y estudiado como un objeto, que ratifica la asimetría" (Ferrara, 1985).
Mishler (citado en Gwyn, 2002) identificó este conflicto empleando la analogía de una lucha de voces. Por un lado, la "voz de la medicina", por el otro la "voz del mundo de la vida". De acuerdo con este enfoque la práctica médica expresa el discurso del modelo biomédico desde "la voz de la medicina", cualquier contribución realizada desde el mundo de la vida es considerada por el profesional como una interrupción. No obstante, cualquier interrupción de la medicina, cuando un paciente está hablando desde su perspectiva de todos los días, no es considerado un entorpecimiento comunicacional desde el orden normativo del modelo de discurso dominante (Gwyn, 2002).

Lo analizado en este apartado da cuenta de los comportamientos afectivoemocionales negativos que generan algunos profesionales, configurando así un escenario de antidialoguicidad y de extensión como modos de generar barreras psicosocioculturales con CCPE.

Postura arraigada quizás en muchos profesionales que probablemente han recibido una formación universitaria basada en modelos clínicos restrictivos a lo biomédico, prescindiendo 0 excluyendo intencionalmente todo lo social, cultural y humano que los completaría con una formación médica integral. Las vigentes, son formas científicamente arcaicas y desfasadas del ejercicio humanamente responsable y científicamente eficaz de esta profesión.

3. Contenidos de las estructuras psicosocioculturales de los profesionales de la salud que dinamizan la barrera: la influencia de la formación universitaria en la relación con consultantes de contextos pobres estructurales

Existen aspectos vinculados a un tipo particular de formación recibida durante las carreras de grado en la Universidad que al contactar con la cultura popular en general $y$, en este caso particular, con los saberes y 
prácticas populares en salud, genera instancias de incomprensión y posibles efectos de reacciones emocionales negativas que disminuyen significativamente la eficacia y eficiencia de la inversión en salud por parte del Estado.

Bernstein (1994) considera que las reglas del discurso pedagógico regulan las formas de conciencia de los estudiantes y las relaciones de poder entre los actores sociales. Muchas veces, este discurso pedagógico reproduce los principios del poder y el control social creando una determinada forma de conciencia de rol 0 aprendizaje en los estudiantes en cuanto a la forma de poner en escena la futura práctica profesional.

$\mathrm{Si}$ a un discurso pedagógico hegemónico, autocrático, verticalista y unidireccional le sumamos, en el caso de la medicina, un modelo clínico-restrictivo, se genera el escenario propicio para que los educandos tiendan a imitar y a reproducir luego acríticamente, en sus prácticas profesionales con las comunidades, ese trato que desde esos modelos docentes y cientificistas deshumanizados les propiciaron a ellos. Al respecto, algunos médicos y médicas expresaban lo siguiente en un estudio previamente realizado (Juárez, 2012):

(...) la facultad a uno lo forma para que sea un robot que no siente, que no piensa, que no nada y la realidad cuando uno está haciendo la facultad, a mi me paso, se te despiertan un montón de sentimientos con pacientes internados $y$ es totalmente normal me parece a mí, me parece lógico también, yo por ahí en la facultad sufrí mucho eso, notaba a los médicos, muy fríos, muy distantes.... (324:331. P19:29 Entrev. C3).

(...) lo que pasa es que uno como profesional, cuál es tu objetivo, mi objetivo es que las personas se sientan mejor $y$ se sientan satisfechas con las cosas que uno les puede brindar, por ahí mis compañeros estaban contentos porque hicieron la súper cirugía de no sé qué, a lo mejor se murió el paciente, pero están contentos porque la hicieron como decía en el libro, los objetivos de ellos son diferentes a los míos, (720:734. P19: 121. Entrev. C3).

Son numerosos los estudios que han indagado en la temática de la formación de estudiantes de medicina y su encuentro con ciudadanos y ciudadanas en el rol de pacientes.

Balint, Balint, Gosling y Hildebrand (1984) planteaban cómo en los entrenamientos médicos los estudiantes son influenciados por modelos docentes cientificistas que se centran en la enfermedad de la persona considerada como "caso o hecho científico". Dyer (1994) denominaba toxic intern syndrome a una reacción defensiva que vivencia el estudiante de medicina y que se manifiesta en cinismo, aburrimiento, insensibilidad e irritabilidad frente a la realidad de sus pacientes.

En la actualidad Kleinman (2011) llama "años precínicos" a los primeros años de la carrera de medicina y "años cínicos" a los de las guardias y las residencias, donde los estudiantes se centran en un plano técnico disociándose de los valores y vivencias emocionales con los pacientes. Tajer (2012) denomina a este tipo de formación como "entrenamiento médico antiempático".

Tal vez, es este el perfil de profesional que necesita el antes mencionado complejo industrial promotor del mercado de la enfermedad, el de un operador 0 dinamizador frío, prescindente de toda empatía, por lo tanto un robot humano abierto a todo tipo de estimulador para desplegar comportamientos autoreferenciados a los propios intereses (prestigio, lucro, satisfacción de la necesidad de dominar, etc.). 
Es interesante vincular estos planteos a la idea de cómo los estudiantes tienden a desarrollar sus prácticas y formación imitando a determinados modelos docentes. D'Ottavio (2001) resalta este aspecto planteando que en los últimos años puede advertirse cómo los alumnos de la carrera de medicina son influidos por un mercado de trabajo que los lleva a pensar a lo largo de la carrera sólo en términos de "especialidad", en consonancia con los modelos docentes que se les han presentado, siendo la gran mayoría de estos docentes especialistas en el ciclo clínico, prescindiendo de la formación en el plano humano y social que supone la práctica médica.

Al respecto uno de los médicos entrevistados expresaba:

\section{(...) la relación médico-paciente la} aprende cada uno a poncho, capa $y$ espada, porque no hay nadie que te venga a decir (...) de habilidades sociales, de que le puede llegar a molestar al paciente, o cómo decir las cosas, (...) nadie te enseña nada .... algunos 10 aprenderán $y$ otros nunca lo aprenden, ese es el drama de la frustración no solo del paciente sino la frustración de los mismos médicos .... (384:391. P10: 32. Entrev. C2).

Lo planteado hasta aquí nos permite advertir cómo la orientación actual en la gran mayoría de las universidades, en lo referido a la formación de los profesionales y técnicos de salud, responde a un paradigma estrictamente clínico restrictivo y deshumanizado, centrado en la enfermedad $y$ en enfoques asistenciales orientados por el modelo de consultorio privado o práctica liberal de la profesión. Se trata del escenario propicio en el que se promueven instancias generadoras de comportamientos que denotan una marcada asimetría y ejercicio de poder en la relación con CCPE, instalándose dispositivos de incomprensión y/o rechazo mutuo.

En escenarios con tales características Vallaeys (2007) plantea que estudiantes y graduados pueden definir vertical y predeterminadamente las "carencias" de las poblaciones y las respectivas "soluciones" a sus dificultades desde procesos sociales que, por omisión 0 predeterminación, terminan implicando una destrucción paulatina de la cultura de las comunidades, generando lo que denomina "etnocidio".

Múltiples ejemplos existen para graficar este fenómeno; el autor menciona el caso de la creación de pozos de agua en el pueblo de Burkina Faso, los que alteraron la habitual rutina de las mujeres de las etnias africanas que allí habitaban y que encontraban su espacio de libertad al salir en grupo a buscar el agua lejos de los varones que dominaban el espacio público del pueblo.

Lo planteado intenta graficar cómo la barrera psicosociocultural se dinamiza y genera sus efectos afectando a poblaciones enteras teniendo, como uno de sus posibles factores, un tipo de formación inadecuada.

Atendiendo a lo desarrollado puede considerarse cómo la formación universitaria recibida, principalmente desde modelos bioclínicos restrictivos y verticalistas, configura los contenidos de las estructuras psicosocioculturales de los profesionales de la salud, conformando las representaciones sociales, los sistemas de significación y sistema valorativo-actitudinales, sistemas de atribución, imágenes, prejuicios, pautas culturales, estilo de desempeño de roles, con que ellos y ellas se enfrentan a las CCPE. Como consecuencia, ello puede traer aparejado la falta de ética y de eficacia de sus acciones al definirlas a priori, centrándose solo en lo clínico-patológico, careciendo de todo tipo de comprensión, diálogo y participación de y con las voces y las narrativas de los y las CCPE.

\section{CONCLUSIONES}

Lo analizado hasta aquí nos permite responder a los interrogantes con que iniciamos este artículo: 
- Respecto a algunos comportamientos propiciados por parte de los y las profesionales de la salud que dinamizan el funcionamiento y estructuración de la barrera psicosociocultural hacia los y las consultantes de contextos pobres estructurales advertimos comportamientos bancarios, de extensión, basados en la imposición de significado, en la cosificación de sus consultantes, en el ejercicio de poder que marca la asimetría de una relación en la que el profesional, se considera asimismo el único poseedor de un saber válido, que, en algunas ocasiones pronuncia desde la violencia simbólica de sus comportamientos a consultantes.

- Respecto a la formación universitaria recibida por los y las profesionales y su impacto en la configuración de contenidos representacionales y comportamentales que propician esta barrera, advertimos la preeminencia de un modelo clínico restrictivo de formación que evidencia un vacío en la formación humanista que se manifiesta en sus propias consideraciones respecto a: a) no encontrarse preparados, al egreso de la universidad, para comenzar un trabajo con consultantes; b) no haber recibido herramientas de formación en la dimensión humana y comunicacional, c) falta de herramientas en habilidades sociales; entre otras. Algunos de los médicos entrevistados reconocen el haber tenido que aprender la dimensión relacional con los consultantes en la misma práctica profesional desde un "aprender haciendo".

Retomando la pregunta inicial: ¿Qué futuro es dable pensar para esta relación y su impacto psicosociocultural? consideramos que, si no se modifican ciertos aspectos de la formación en medicina junto con las presiones de grandes corporaciones médicas y farmacológicas jamás el campo de la salud se podrá humanizar, ni se hará eficaz, eficiente y de calidad en pos de una sociedad sana.

\section{PROPUESTA}

Posibilidades de una instancia de encuentro facilitadora entre profesionales de la salud y consultantes de contextos pobres estructurales

Hubo un médico inglés, allá por el siglo XVII XVIII, Sydenham, Io llamaban el Hipócrates inglés. Cuentan que va un chico y le dice: "Maestro me acabo de recibir, ¿qué libro me recomienda para perfeccionarme? Y el gran maestro le dice "Lea El Quijote. Vaya a la humanidad y vuelva". Es decir, lo que falta es el estudio de las humanidades, del hombre. En la facultad la enfermedad es un iceberg, entonces arriba lo visible: la enfermedad biológica. Pero abajo, está el padecimiento, que es la experiencia social del paciente. Por 10 de arriba actuamos con eficacia biológica, abajo actuamos con la eficacia simbólica. La eficacia simbólica actúa con los mismos intermediarios inmunohistoquímicos que la eficacia biológica. Si solamente atendemos lo de arriba del iceberg ¿sabes en que nos convertimos? Y lo digo con respeto porque yo también lo pasé, somos plomeros del cuerpo. Tenemos que hacer las dos medicinas: la del cuerpo y la del espíritu. Una sola de las dos es una medicina hemipléjica. [Se añaden las itálicas] (Entrevista al Dr. Francisco Maglio, 2007).

Lo planteado nos permite visibilizar un horizonte complejo en la relación entre médicos/médicas y CCPE donde la posibilidad de desactivar los procesos y 
dinámicas que dan origen a la barrera psicosociocultural requiere asumir desafíos que van en tres dimensiones:

1. Formación y entrenamiento médico (extracurricular) orientado al desempeño de comportamientos dialógicos y prosociales en sus relaciones con CCPE que se atienden en el Primer Nivel de Atención de la salud.

2. Reorientación de las estrategias de formación en las residencias médicas. Se trata de promover una capacitación humanista en habilidades y prácticas sociales, orientada a la formación de un profesional relativista cultural que respete los saberes de la población con respecto a la salud.

3. Promover las transformaciones curriculares necesarias a los fines que las carreras de medicina de las universidades públicas generen profesionales dotados de capacidad empática con la población, formados en Ciencias Sociales (antropología cultural, psicología social y comunitaria, sociología, psicología sanitaria, pedagogía dialógica) de modo que se constituyan en profesionales del Primer Nivel de Atención prosociales, dialógicos, cooperantes y protectores de la salud de la población, ayudando a resolver sus fuentes de sufrimiento $y$ circunstancias de vida.

Consideramos que la esencia de una concepción hipocrática que tiene en el médico la figura de un maestro y un amigo de la población que acude a él en búsqueda de ayuda o colaboración para resolver sus problemas de salud o sus temores, es el eje para promover instancias de puentes facilitadores de la relación en la actualidad y en el futuro de la misma.

\section{REFERENCIAS}

Balint, M.; Balint, E.; Gosling, R y Hildebrand, P. (1984). La capacitación psicológica del médico. Evaluación de los resultados de un programa de posgrado para médicos clínicos. Barcelona: Gedisa.

Bernstein, B. (1994). La estructura del discurso pedagógico. Clases, códigos y control. (Volumen IV). Madrid: Morata.

D'Ottavio, A. (2001). Ser médico. Reflexiones sobre la formación y la práctica médicas. Santa Fe: Homo Sapiens Ediciones.

De Lellis, M., Álvarez, M., Rosetto, J. y Saforcada, E. (2006). Psicología y políticas públicas de salud. Buenos Aires: Paidós.

Emmanuelle, E.S. (1998). Educación, salud, discurso pedagógico. Buenos Aires: Novedades educativas.

Entrevista a Francisco "Paco" Maglio. (2007) Serie Foto-reportajes de IntraMed. Versión de texto. Recuperado el 28 de mayo de 2010, de: http://www.intramed.net

Escotorin, P; Juárez, M.P; Cirera, M. y Roche-Olivar, $\quad$ R (2011). La prosocialidad: una vía segura para construir relaciones de confianza con los pacientes. En P. Escotorin y R. Roche Olivar (Eds.). Cómo y por qué prosocializar la atención sanitaria: reflexiones, desafíos y propuestas. (pp. 22-36). Barcelona: Fundación Martí L'Humà.

Feldman- Stewart, D; M.D. Brundage y C. Tishelman. (2005). A conceptual framework for patient-professional communication: an application to the cancer context. Psycho-Oncology. (14), 801-809. Recuperado el 12 de noviembre de 2008 de: www.interscience.wiley.com

Ferrara, F. (1985). Teoría Social y Salud. Conceptualización del campo de la salud. Buenos Aires: Catálogos.

Freire, P. (1973). Pedagogía del Oprimido. (10 ed. en castellano, 1970). Buenos Aires: Siglo XXI.

Gwyn, R. (2002). Power, asymmetry and decision making in medical encounters. En R, Gwyn. (Ed.): Communicating health and illness (pp. 61-91). London: SAGE Publications.

Hammersley, M y Atkinson, P. (1994). Etnografía. Métodos de investigación. Barcelona. Buenos Aires. México: Paidós. 
Izquierdo C. (1996). La reunión de profesores. Participar, observar y analizar la comunicación en grupo. Barcelona: Paidós.

Juárez, M.P (2009a). Psicopedagogía y salud: una experiencia comunitaria en Río Cuarto. Tesis de Maestría no publicada. Maestría en Ciencias Sociales. Facultad de Ciencias Humanas, Universidad Nacional de Río Cuarto.

Juárez, M.P (2009b). Comunicación de Calidad Prosocial desde la perspectiva del paciente en la comunidad barrial Hipódromo, Argentina. Tesina de Suficiencia Investigadora no publicada. Doctorado Psicología de la Comunicación: Interacción Social y Desarrollo Humano. Universidad Autónoma de Barcelona.

Juárez, M.P (2012). Relación médicoconsultante en el Primer Nivel de Atención del Subsistema Público de Salud. Estudio de casos y propuesta de formación médica en pedagogía dialógica. Tesis de Doctorado en Psicología (no publicada). Universidad Nacional de San Luis. Argentina.

Kalinsky B. y W. Arrúe. (1996). El conflicto de creencias en la relación médico/paciente. ¿Un equívoco adrede?. En B. Kalinsky y W. Arrúe (Comps.): Claves antropológicas de la salud. El conocimiento en una realidad intercultural. Buenos Aires: Miño y Dávila.

Kleinman A. (2011). The divided self, hidden values, and moral sensibility in medicine. Lancet. (377). 804-805.

Lasso Toro, P. (2012). Atención prenatal: ¿tensiones o rutas de posibilidad entre la cultura y el sistema de salud? Pensamiento Psicológico, 10 (2), 123133.

Maslow, A. (1991). Motivación y personalidad. Madrid: Díaz de Santos.

Max-Neef, M.; Elizalde, A. y Hopenhayn, M. (1986). Desarrollo a escala humana. Santiago de Chile: Cepaur -Fundación Dag Hammarskjold.

Mendoza González, B.Z. (1999). La relación médico-paciente en pueblos indígenas: algo más que la búsqueda de la curación. Salud Problema. (4), 7. Recuperado el 16 de marzo de 2007, de: http:www.xoc.aum.mx/aum/publicaciones/r evistas/saludproblema/pagina/texte/sietef.p df

Morales Calatayud, F. (1999). Introducción a la psicología de la salud. Buenos Aires, Barcelona, México: Paidós.

Neiman, G. y Quaranta, G. (2006). Estudio de caso en la investigación sociológica. En: Vasilachis de Gialdino, I (Ed.) Estrategias de investigación cualitativa. Barcelona: Gedisa, 213-237.

Saforcada (2002b). Formación universitaria de los profesionales y técnicos de la salud. Los psicólogos. En E. Saforcada (2002). Psicología sanitaria. Análisis crítico de los sistemas de atención de la salud. (pp. 167185). Buenos Aires: Paidós.

Saforcada, E. (2001). El factor humano en la salud pública. Una mirada psicológica dirigida hacia la salud colectiva. Buenos Aires: Proa XXI.

Saforcada, E. (2002a). Psicología sanitaria. Análisis crítico de los sistemas de atención de la salud. Buenos Aires: Paidós.

Saforcada, E. y Colaboradores del Proyecto Intracomunitario de Neurodesarrollo San Luis (PINSAL). (2011). La estrategia de mínimo operante en el desarrollo salubrista de comunidades. Universidad Nacional de San Luis. San Luis: Nueva editorial universitaria.

Saforcada, E; de Lellís, M y Mozobancyk., S. (2010). Psicología y salud pública. Nuevos aportes desde la perspectiva del factor humano. Buenos Aires: Paidós.

Seligman, M.E, P. (1989). Indefensión. En la depresión, el desarrollo y la muerte. Madrid: Debate.

Stake, R. (1998). La investigación con estudio de casos. Madrid: Morata.

Strauss, A. y J. Corbin (1998). Fundamentos de investigación cualitativa. Técnicas y procedimientos para desarrollar Teoría Enraizada. Thousand Oaks London New Delhi: Sage Publications.

Tajer, C (2012). La formación cultural y humana del médico. Una propuesta práctica. Recuperado el jueves 26 de enero de 2012 de:

http://www.intramed.net/contenidover.asp? contenidoID=73791\&uid=505856\&fuente=i news

Thompson, T. L. (1990). Patient health care: issues in interpersonal communication. En: E. Berlin Ray y L. Donohew (Comps): 
El problema de la barrera psicosociocultural interpuesta entre los profesionales de la salud y consultantes de contextos pobres estructurales. Reflexiones sobre una cuestión compleja

Communication and Health.Systems and applications. Jersey, Hillsdale: LEA.

Vallaeys, F. (2007). El difícil camino hacia un desarrollo social ético. Recuperado de: http://www.encuentroscj.org/facipub/upload /cont/813/cont/file/el-dificil-camino-haciaun-desarrollo-socia-etico.pdf

Vidal y Benito, M.C (2010). La relación médico-paciente. Bases para una comunicación a medida. Buenos Aires: Editorial Lugar. 